\title{
Using Mobile Phone Sensors to Detect Rapid Respiratory Rate in the Diagnosis of Pneumonia
}

\author{
Xingjuan Li
}

\begin{abstract}
In resource-poor areas, the rapid respiratory rate is an important factor to determine pneumonia. Identifying the rapid respiratory rate from robust sensors can solve the problem of labor-intensive practice of counting respiratory rate for doctors. In this paper, the smart phone sensors are proposed to detect physical signs of breathing in a non-invasive way. The proposed method was targeting at detecting the rapid respiratory rate. It was designed by android apps with the ability to acquire breathing signals from phone sensors, and follow-up biomedical signal processing. Data recovery is used to reconstruct the whole information of collected data from android. Moreover, based on the features of breathing signal, additional signal processing is used to reduce interference. Finally, the rapid respiratory rate is measured by comparing with the definition of rapid respiratory rate given by the World Health Organization. Smart phone provides a diagnostic platform for suspected pneumonia patients. The whole algorithm may give an error of \pm 2 breaths per minute. Therefore, this research provides a new way of capturing breathing signal using phone sensors with high performance. Using this proposed technology, the danger signs of pneumonia can be recognized earlier, thus more lives will be saved by prompt treatments.
\end{abstract}

Index Terms-Rapid respiratory rate, pneumonia, mobile phone sensors, bio-medical signal processing.

\section{INTRODUCTION}

Pneumonia is a common disease encountered by physicians, and it is also the leading killer among children around the world. There are about 2 million children dying from pneumonia every year, which is more than AIDS, malaria and measles combined [1]. Pneumonia can be caused by many reasons, for example, bacteria, fungi and viruses. The common clinical symptoms of pneumonia in children under-five include rapid or difficult breathing, lower chest wall in-drawing [2], cough, fever, chills, headaches, loss of appetite and so on. In resource-poor settings, tachypnea, cough and fever are used as the standard of diagnosing pneumonia. Although there are effective treatments of pneumonia, only a few are available to children in remote areas. According to a survey, about 600,000 lives can be saved if antibiotics were delivered promptly to children with pneumonia [3].

Mobile phones are used everywhere, even in remote regions [4]. Their capabilities are far beyond working as a communication tool. Mobile phone sensors such as accelerometers and gyroscopes are good enough to monitor physical signs caused by breathing activity. The operating

Manuscript received December 14, 2014; revised March 1, 2015.

Xingjuan Li is with the School of Information Technology and Electrical Engineering, University of Queensland, Brisbane, QLD 4072 Australia (e-mail: mirandalxj@gmail.com) system of a smart phone, such as android and iOS, can read and record the data recorded by phone sensors. These data can be further processed by matlab to identify rapid respiratory rate. It is shown that a rapid respiratory rate together with presenting cough is a reliable basis for diagnosing non-severe pneumonia in children under-five [5].

Some methods have been proposed to monitor respiratory rate. One of the methods is analyzing the pressure wave caused by the oxygen passing though a magnetic field reflects the oxygen pressure [6]. Another method is using pulse oximeters, which measures haemoglobin saturation in arterial blood. Spirometer is a method that measures the changes on volume of gas [7]. Assessing arterial carbon dioxide concentration from exhaled gases can also reflect respiratory rate. These methods focus on gas delivery and gas exchanges in arteries. They require special equipment and professional operation, which are limited in resource-poor regions due to lack of healthcare workers. Also, these techniques give an error of 2 to 4 breaths per minute.

I propose a reliable, efficient, inexpensive and simple method to detect rapid respiratory rate with the use of smart phone sensors in a non-invasive way. It is based on the characteristic features of breathing signals. Breathing causes chest and abdominal movements, which can be detected by physical sensors on the smart phones. The signals detected by phone sensors can reflect breathing activity well after processing. The proposed method has been tested on six adults with high performance in determining respiratory rate.

\section{METHODS}

The whole system consists of three parts (see Fig. 1). One is android app, which can read the recorded data from the sensors. One is data processing part, which is used to remove noise from breathing signal. Another is user feedback part, which can give a report to the user.

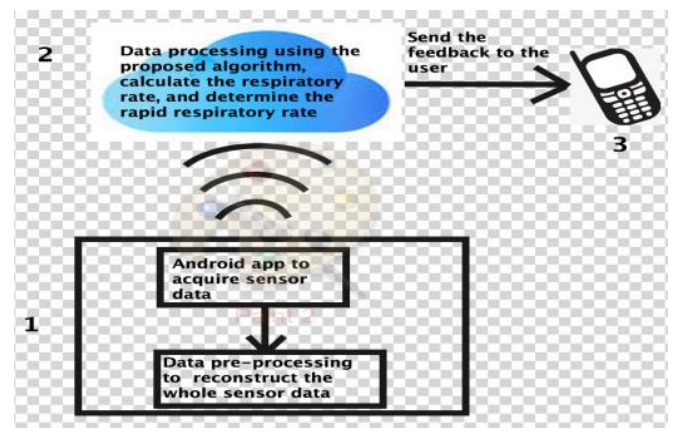

Fig. 1. The components of the whole system.

\section{A. Develop Applications}

An android accelerometer app and the gyroscope app were developed separately to access accelerometers and 
gyroscopes in the smart phone. The reason for using different apps for different sensors is that android uses different timestamps to read sensor events for different sensors [8]. The timestamp controls the speed of android to read a sensor event. The application is made up of three user-interfaces, namely data reading interface, sensor capability interface, and a canvas to describe sensor values (see Fig. 2). Android reads the sensor values in a three-axis coordinate system. The coordinate system is defined relatively to the screen with the $\mathrm{x}$-axis representing horizontal position, the $\mathrm{y}$-axis representing vertical position, and the $\mathrm{z}$-axis pointing towards the outside of the screen [9]. The sensor values were recorded through android. Android is a convenient user operating system. It provides 4 types data delay, which can be used to control the time interval at which sensor events are sent to the application. In this experiment, a 20,000-microsecond delay was used in both accelerometer and gyroscope apps. There is a minimum delay specified by the manufacturer, which controls the maximum speed to read discrete values from the analog signal. For the accelerometer in HTC one, the minimum delay is 10,000 microseconds. And for the gyroscope, the minimum delay is 1,200 microseconds. The data delay used in the app cannot go below this minimum delay. The resolution of accelerometer and gyroscope are 0.038307227 and 0.0012217305 respectively. The phone can be placed in front of the chest or abdomen to detect the movement caused by breathing.

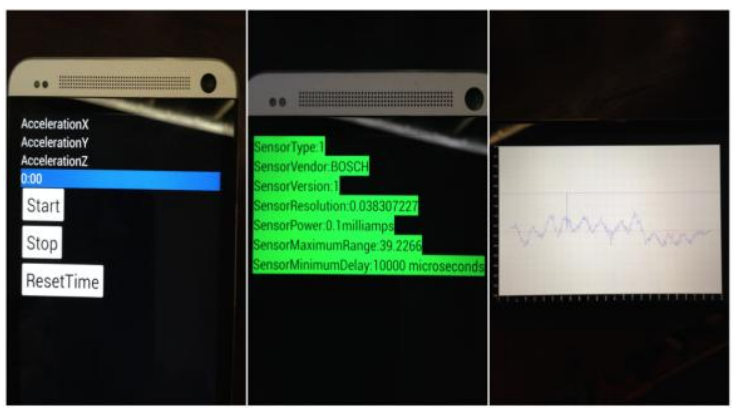

Fig. 2. Android accelerometer app used for data acquisition.

\section{B. Data Acquisition and Pre-processing}

The phone sensors convert the analog signals to discrete signals. Android reads the sensor events from the sensors according to its rules. The rule used in android is that it only reads the values changed in the parameters it is measuring. Therefore, some information would be lost in the process of data acquisition. In order to retrieve the lost information, the system time was recorded when a sensor value was sent to the application. The system time is a standard clock expressing milliseconds [9]. The system time together with the data delay specified to send sensor values to the app were used to identify the time and location the information were lost. This is because the lost information was the same as the previous values in the parameters android was measuring. This would mean that the whole information was reconstructed from the time and location (see Fig. 3).

\section{Data Processing}

The data processing part focused on computing breathing rate.

1) The reconstructed data were processed under a moving average filter of window size 10 to remove small spikes.
Then, a Gaussian filter of window size the same as the data delay was introduced to filter the data. After filtering, the trend of the signal was kept while most interference was removed (see Fig. 4). The design of average filter and Gaussian filter was based on the following formulae:

$$
\begin{gathered}
y[i]=\frac{1}{M} \sum_{j=0}^{M-1} x[i+j] \\
g(x)=\sqrt{\frac{a}{\pi}} \cdot e^{-a \cdot x^{2}}
\end{gathered}
$$

2) The peak-peak time interval (duration) was then calculated based on the filtered data by the following formulae:

$$
\begin{gathered}
P P=k(i+1)-k(i) \\
\text { Duration }=\frac{P P}{F s}
\end{gathered}
$$

where, $p p$ is the interval between two consecutive peak values, $F s$ is the data delay.

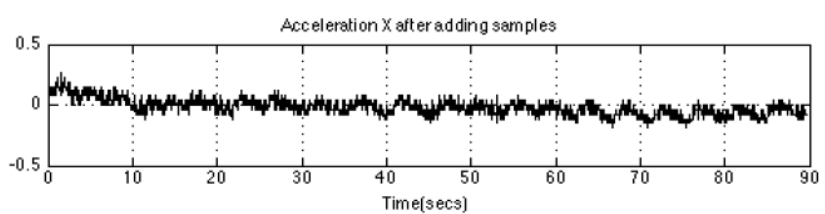

Acceleration $Y$ after adding samples

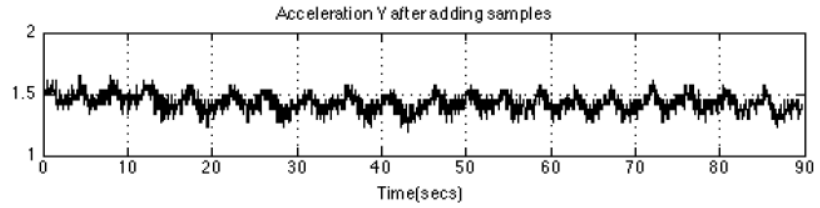

Acceleration $Z$ afteradding samples

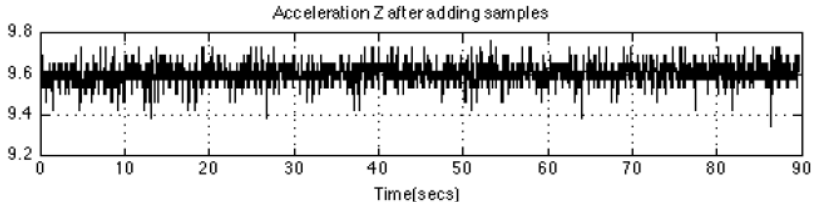

(a)

Gyroscope $X$ after adding samples

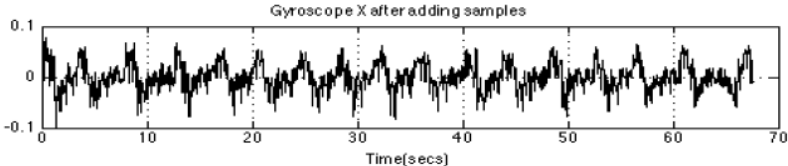

Time[secs)
Gyroscope $Y$ after adding samples

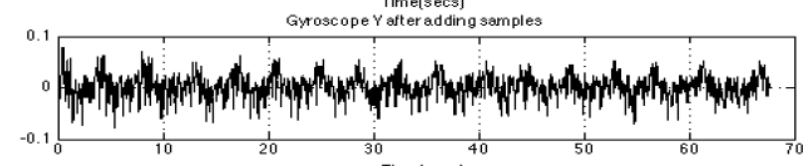

Timessecs)
Gyroscope $Z$ afteradding samples

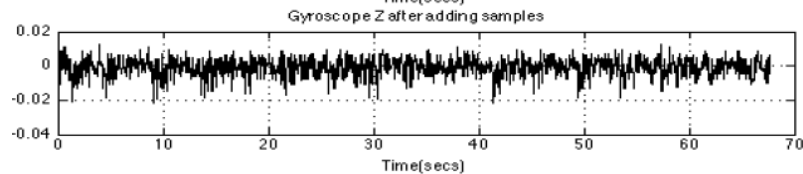

(b)

Fig. 3. Reconstructed accelerometer data (a) and gyroscope data (b) measured from abdomen.

3) Calculate the maximum, minimum and mean of the duration. The average of maximum-mean and minimum-mean were used as the reference to remove noise.

4) Finally, compute the mean duration over three-axis and use the minimum value as the final duration. The final duration was then used to calculate breathing rate in one minute.

$$
B R=\frac{60}{\text { Duration }}
$$


The data processing algorithm can be uploaded on cloud for users. After users measure their breathing activity, the measured data will be transferred to the cloud for further data processing. The result will be sent back to the user.
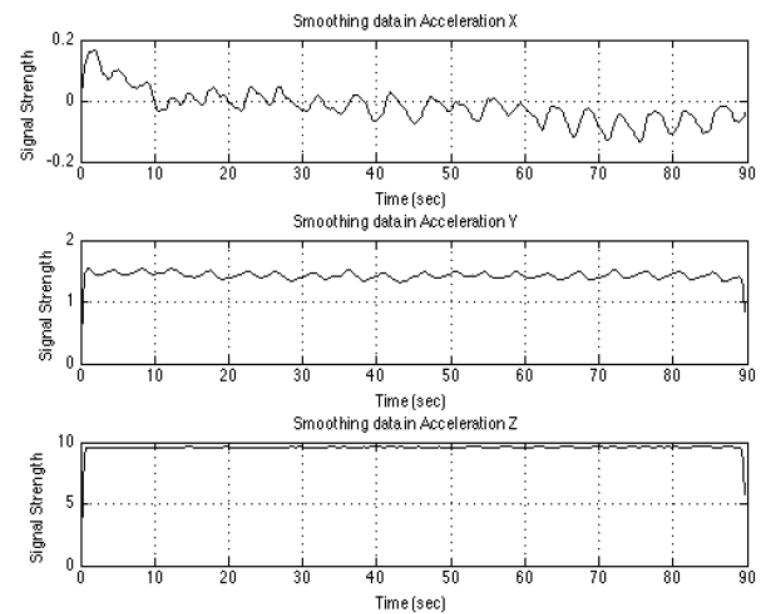

(a)
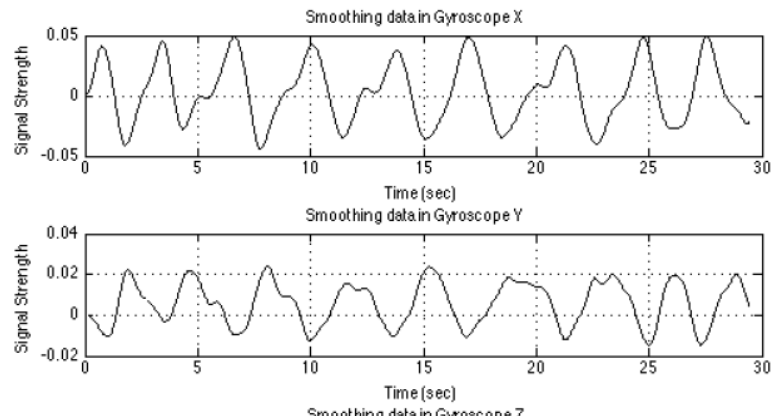

Smoothing datain Gyroscope $Z$

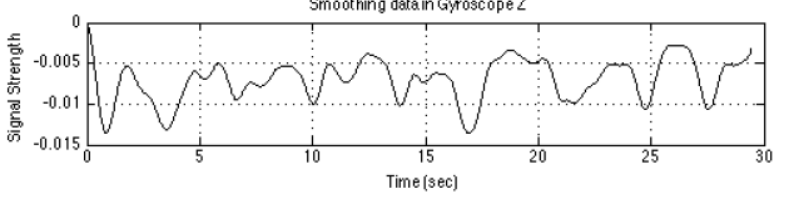

(b)

Fig. 4. Smoothed accelerometer data (a) and gyroscope data measured from abdomen (b).

\section{Determine Rapid Respiratory Rate}

According to the World Health Organization, a rapid respiratory rate is defined according to the age and respiratory rate [10]. For children less than 2 months, a rapid respiratory rate is higher than 60 breaths per minute. For children between 2 months and 1 year, a rapid respiratory rate is higher than 50 breaths per minute. For children from 1 year to 5 years, a rapid respiratory rate is higher than 40 breaths per minute. For an adult, a rapid respiratory rate is higher than 20 breaths per minute.

In the last step, the computed breathing rate was compared with the definition of rapid respiratory rate given by the World Health Organization. If the patient presented a rapid respiratory rate and cough, he or she is presumed to have pneumonia. Further treatment, like antibiotics, can be provided promptly.

\section{EXPERIMENTAL RESULTS}

The proposed method has been tested on six people (ranging from 22 to 52 years) using accelerometer and gyroscope. The smart phone (HTC one) was placed on chest or abdomen in its default position. The tested results were shown in Table I.

\begin{tabular}{|l|l|l|l|}
\multicolumn{5}{c|}{ TABLE I: EXPERIMENTAL RESULTS } \\
\hline Position & Sensor type & $\begin{array}{l}\text { Real } \\
\text { respiratory rate } \\
\text { per minute }\end{array}$ & $\begin{array}{l}\text { Tested } \\
\text { respiratory rate } \\
\text { per minute }\end{array}$ \\
\hline Abdomen & Accelerometer & 13 & 13 \\
\hline Chest & Accelerometer & 10 & 10 \\
\hline Chest & Gyroscope & 9 & 11 \\
\hline Abdomen & Gyroscope & 15 & 16 \\
\hline
\end{tabular}
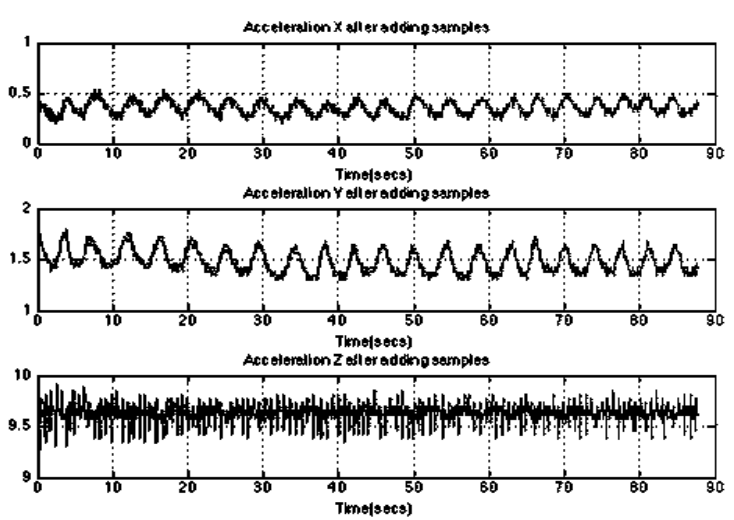

(a)

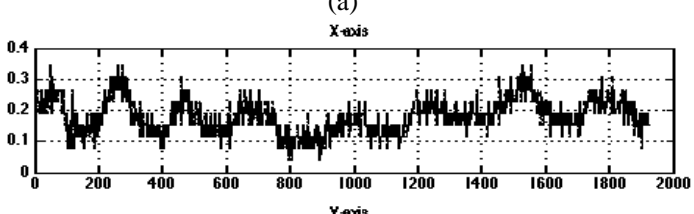

Yexis

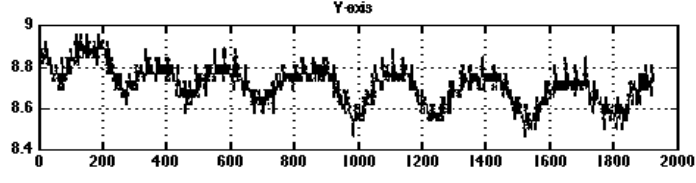

Zwovis

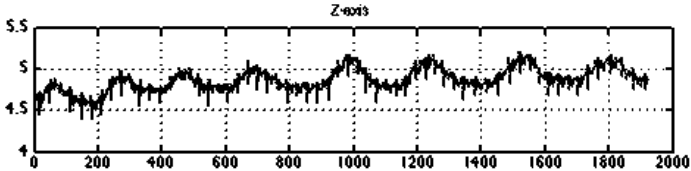

(b)

Fig. 5. Reconstructed accelerometer data measured in front of abdomen (a) and chest (b)

For the data measured by accelerometer from abdomen (Fig. 5(a)), the most reliable channels were $\mathrm{x}$ - and y-channel, which measured the signal from phone's and horizontal and vertical position. The tested result showed that the acceleration force on $\mathrm{x}$ - and $\mathrm{y}$-channel changed significantly and revealed the breathing. Although the z-channel, which measured the signal towards the phone's outside, detected the movement of abdomen on its direction, it was contaminated by too many noises. Also, the acceleration force on z-channel was almost stable. However, the most reliable channels were $\mathrm{y}$ - and z-channel when the phone was placed on the chest. The signal acquired by different channels was recorded according to android three-axis coordinate system. The strength of the signal and the position of sensors in the phone also affected the accuracy of different channels. Therefore, in terms of detecting breathing signal by accelerometer, the most reliable channel was y-channel.

For the data measured by gyroscope, they can reveal breathing after processing (see Fig. 6). But they were contaminated by too many noises on all channels. The 
rotation force was affected by many factors, such as breathing and heartbeats.

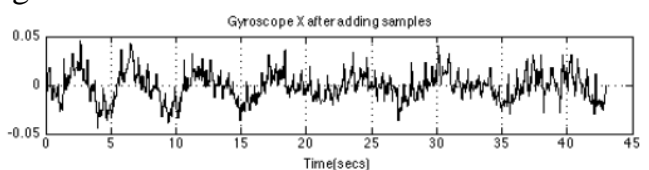

Girroscope $Y$ afteradding samples

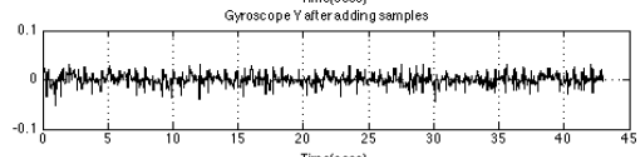

Time(secs)
Gyroscope Z after adding samples

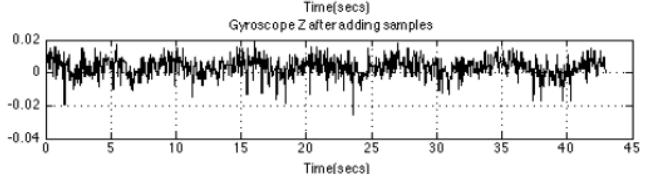

(a)
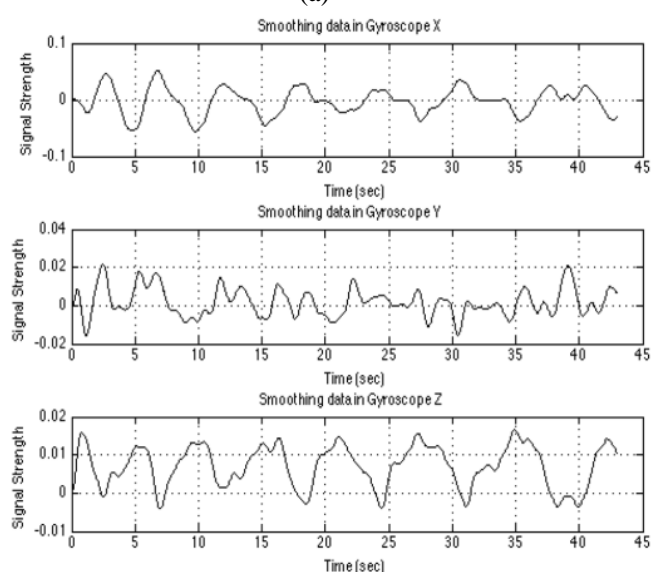

(b)
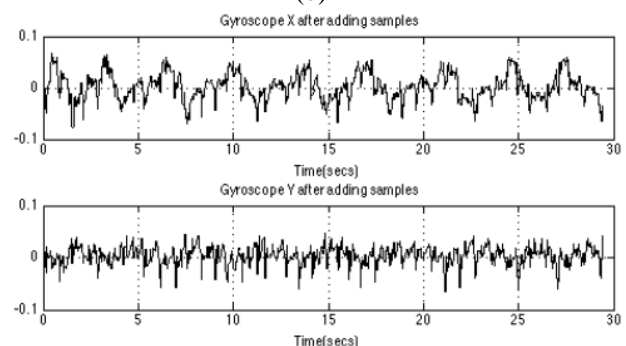

Time(secs)

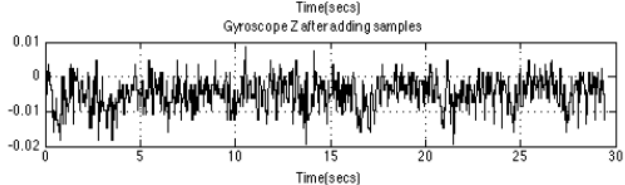

(c)
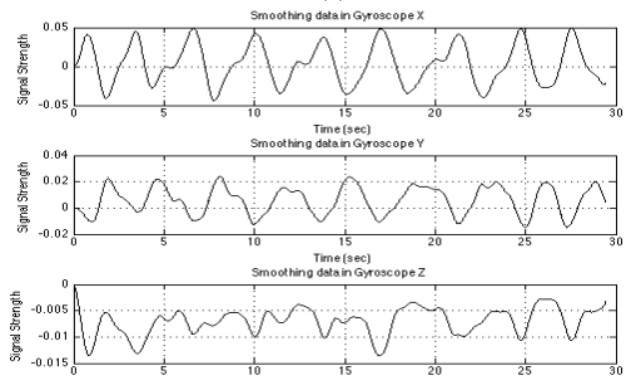

(d)

Fig. 6. Reconstructed gyroscope data and smoothed gyroscope data measured in front of chest (a) \& (b) and abdomen (c) \& (d).

\section{CONCLUSION}

The paper is devoted to providing a convenient solution to overcome the difficulties in diagnosing pneumonia, especially for children under-five living in remote areas, by measuring breathing rate with the use of a smart phone. The proposed algorithm has the ability to determine rapid respiratory rate within 1 minute. In addition, the accuracy of this method is quite attractive, with an error of only \pm 2 breaths per minute. Probably the most attractive point of smart phone usage lies in its convenience as a medical device. However, the proposed method does have some limitations. The smart phone sensors directly measure signal from its environment. If the phone is not fully placed on human body surface, the sensor cannot detect any useful information. Also, in the real field, young children will not be easily kept quietly in the data acquisition process. This would be a problem because any movement can lead to errors in the recorded data. Essential future work will be required to improve the proposed method for diagnosing pneumonia.

\section{REFERENCES}

[1] T. Wardlaw, E. Johansson et al., Pneumonia: the Forgotten Killer of Children, World Health Organization press, 2006.

[2] M. Chisti and M. Salam, "Predictors and outcomes of hypoxemia in severely malnourished children under five with pneumonia: A case control design," Plos One, vol. 8, pp. 1-5, January 2013.

[3] G. Jones, R. Stekette, R. Black, Z. Bhutta, and S. Morris, "How many child deaths can we prevent this year," The Lancet, vol. 362, pp. 65-71. July 2003.

[4] G. Woodrow. (October 2011). Low cast diagnostics based on robust sensors and mobile phones. Australasian Biotechnology. 21 (3). pp. 15. Available: [Online]. http://search.informit.com.au.ezproxy.library.uq.edu.au/documentSu mmary; $\mathrm{dn}=125016846376274$

[5] F. Shann, K. Hart, and D. Thomas. "Acute lower respiratory tract infections in children: Possible criteria for selection of patients for antibiotic therapy and hospital admission," Bulletin of the World Health Organization, vol. 62, pp. 749-753. May 1984.

[6] J. Dorsch and S. Dorsch, Understanding Anesthesia Equipment, 5th ed, Lippincottt, Williams \& Wilkins Press, 2005, pp. 23-25.

[7] G. Morgan and M. Mikhail, Clinical Anesthesiology, 4th ed, Mcgraw Hill Press, 2006, pp. 44-90.

[8] I. Zliobaite and J. Hollmen, "Mobile sensing data for urban mobility analysis: a case study in preprocessing," in Proc. the EDBT/ICDT 2014 Joint Conference, 2014, pp. 309-314.

[9] Android Developers. Available: [Online] http://developer.android.com/reference/android/hardware/SensorEven t.html

[10] A. Pio, "Standard case management of pneumonia in children in developing countries: The cornerstone of the acute respiratory infection programme," Bulletin of the World Health Organization, vol. 81,2003 , pp. 298-300.

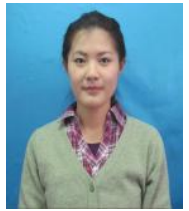

Xingjuan Li was born in 1990. She received her master's degree in electrical engineering from University of Queensland. Brisbane, Queensland, Australia, in 2014. Her research interest is in bio-medical engineering. 